\section{VIRTUAL ENVIRONMENTS FOR THE TREATMENT OF CLAUSTROPHOBIA}

\author{
C. Botella and S. Quero, \\ Jaume I University, Spain \\ C. Perpiñá and R.M. Baños, \\ University of Valencia, Spain \\ M. Alcañiz, J.A.Lozano, and A. Rey \\ Polytechnic University of Valencia, Spain
}

Editor: Max M. North

\begin{abstract}
Virtual Reality (VR) is a new technology that is having a great impact on various areas of health science. The use of VR is of special interest in the treatment of psychological disorders. Reasonable data exist on the effectiveness of this technology in the treatment of different anxiety disorders. The present paper explains in detail a psychological program for the treatment of claustrophobia using VR, whose effectiveness has already been demonstrated. [Botella et al.,1998]. In addition, we describe the technical aspects of the software and point out several limitations VR still presents in the area of psychological treatment. Color images and video clips are included with the CDROM version.
\end{abstract}

\section{Introduction}

Since the beginning of the 1960s the international scientist community has been working in the virtual reality (VR) field, and has recognized this tool as a highly powerful man-computer interface with broad technical and scientific applications. The clinical field also recognizes the enormous potential presented by the technology. Several research groups have already shown its benefits and possible applications. According to Greenleaf [1995], a pioneer in the application of advanced technologies in the health field, VR currently presents applications in numerous areas, including simulation and planning of surgical procedures, therapy, diagnosis, education, telemedicine, rehabilitation, architectural design of sanitary devices, and so on. The potential of VR in the clinical disciplines is clear. For instance, in August 1997 an entire issue of the journal Communications of the ACM was devoted to current developments and future possibilities of VR in the health and psychological treatment and rehabilitation fields.

\section{Virtual Reality and Psychological Treatments}

The applications of VR in the psychological treatment field are currently in their infancy. The following represent a overview of pioneering studies:

1. Antecedents. An antecedent is the case of special glasses which alter the depth perception, used by Schneider [1982] in order to magnify the height sensation during the process of in vivo exposure. Antecedent works that recommend the future use of VR for psychological treatments include [Tart, 1991] in general, and [Knox, Schacht and Turner, 1993] for performance anxiety.

2. Spiders Phobia. A group at the University of Nottingham has developed a VR system for the treatment of a specific phobia, the spiders phobia. The patients wear a Head Mounted Display (HMD) by means of which a virtual spider can be "visualized". Its realism is gradually increased until the patient's tolerance level allows him/her to cope with it in the real world [Vince, 1995]. The group of Hoffman [Carlin, Hoffman and Weghorst, 1997] reveals, in a case study, the utility of a VR application which consists of software for the treatment of the spiders which is used in conjunction with augmented reality techniques. These authors report on the success achieved in a patient whose phobia was extremely limiting.

3. Acrophobia. The Kaiser-Permanent Medical Group of California has developed an experimental system to assess the utility of VR for the treatment of acrophobia. In this system the patient must pass trough a deep gully by crossing a suspension bridge and a narrow board. This system was used with 32 patients and resulted in a $90 \%$ success rate. Dr. Lamson, responsible for this project, states that the patients have the sensation of having coped with this fear and overcome it. In his own words: "it is an excellent tool which allows the patient to build a strong sensation of confidence" [Vince, 1995]. The group of Rothbaum and North of Clark Atlanta University has published the first reports (a case study and a controlled study) on the utility of software they designed for the treatment of acrophobia [Rothbaum, Hodges, Kooper, Opdyke, Williford and North, 1995a b; North and North, 1994; North and North, 1996]. In 1992 they developed the VREAM (Virtual reality development software package and libraries) software to generate a virtual environment for the treatment of acrophobia. They created a setting with an external lift which is adjusted to different heights with the patient being placed on a balcony of each floor. The patient pointed out that he felt a high degree of immersion in the virtual environment, and within 8 sessions he felt relaxed in a height level corresponding to the fifteenth floor.

4. Fear of flying. This same group has designed software for the treatment of fear of flying. In a case study [Rothbaum, Hodges, Watson, Kessler and Opdyke, 1996] they report on the successful utilization of the system. The treatment 
was carried out during 6 sessions of approximately 35-45 minutes. The patient was a 42 year old woman suffering from a serious fear of flying.

5. Agoraphobia. The North group [Rothbaum et al., 1995a] has carried out work on agoraphobia by creating agoraphobic environments and testing them with students. The subjects exposed to the virtual exposure improved in a significant way, whereas changes were not observed in the control group.

6. Claustrophobia. Our own group has designed software for the treatment of claustrophobia, and we can already affirm that the claustrophobic context is able to activate a high degree of anxiety in the patients. Moreover, the patients can overcome the phobia by means of the virtual exposure [Botella, Baños, Perpiñá, Villa, Alcañiz and Rey, 1998; Botella, Perpiñá, Baños, Ballester and Quero, in press]. In order to complete the information regarding to works which are being carried out on the treatment of anxiety disorders by means of VR, we shall resort to the recent paper of Strickland, Hodges, North and Weghorst [1997]. These authors report on the latest projects being carried out, though most of them are still in progress and the results yet unpublished.

7. Post-traumatic stress. Hodges and Rothbaum have begun to use VR for the treatment of post-traumatic stress disorder. Virtual scenarios that reproduce the images of the war are being used to treat Vietnam war veterans.

8. Fear of speaking in public. The North group at Clark Atlanta University is studying the utility of VR for the treatment of fear of speaking in public. The subjects see themselves immersed in a virtual scenery. When they start speaking they have an audience in front of them which is adjusted gradually to a clearer and more realistic scene.

9. Fear of driving. A controlled study is being initiated to determine the utility of VR for the treatment of fear of driving. Currently no data from the study has been published.

The first book focused solely on VR psychotherapeutic treatments has been recently published: "Virtual reality therapy: An Innovative Paradigm" [North, North, and Coble, 1997]. In it additional programs for the treatment of agoraphobia and fear of flying in combat are described. The first edition of the book has rapidly gone through a first printing, and a second volume is about to appear.

In summary, even though the number of studies is small, there exists clear evidence of the utility of VR for the treatment of specific phobias. However, it is also clear to us that some problems still exist, the most important being the following [Botella, et al., 1998]:

1. It is necessary to extend the application fields even further and confirm whether VR is effective in other more general psychological disorders.

2. Many of the research reports are focused on mere case studies, which follow only a simple $\mathrm{AB}$ design, with consequent generalization problems deriving from this kind of design.

3. Most of the treated patients are subclinical subjects, that is, people whose problem is not so serious or so troublesome to ask for psychological treatment.

4. For all we know, there only exists one published controlled study [Rothbaum et al., 1995a], and although a waiting list control group was used, there was not a placebo group.

5. The published works so far only offer pre-treatment versus post-treatment data; they do not report anything regarding to recovery, stability, post-therapy advances, relapses and so on. It is, therefore, absolutely necessary to obtain follow-up data.

6. In some works a "pure" VR treatment has not been applied. For example, in the study of Rothbaum et al., [1996] on fear of flying, techniques for the management of anxiety were previously used (cognitive restructuration, thought stopping, and active relaxation) with the subsequent pulling effect of the treatments, causing difficulty in the interpretation of the results.

7. Given that the works published up to now are focused on anxiety disorders, it is also necessary to point out the lack of comparison between achieved effectiveness with VR and that achieved by the current non-VR treatment. Specifically, it would be well to know to what degree VR is effective if we compare it to "in vivo" exposure or to imagination exposure.

\section{VR Treatment for Claustrophobia}

As it is concluded in the works reported up to now, evidence on the utility of VR for the treatment of specific phobias has started to appear. However, it is necessary to go on obtaining data regarding to the effectiveness of this new tool and to confirm whether it is also effective in other psychological disorders. In this work we shall focus on claustrophobia.

The choice of claustrophobia is justified by the special characteristics of this phobia. Such characteristics have made it be considered as functionally equivalent to agoraphobia, but with a smaller rank of avoidance [Barlow, 1988; Öst, 1987; Booth and Rachman, 1992]. Moreover, claustrophobia, by itself, may be very limiting and cause a notable impairment in life since closed places are commonly experienced in daily life (lifts, planes, tunnels, cabins, medical diagnosis procedures such as CAT scan, MRI, etc.)

\subsection{PSYCHOLOGICAL ASPECTS}

In the design of the software special attention was paid not only to the general guidelines which should be followed in the treatment of this problem but also, and in a very special way, to the different elements that might facilitate the immersion and the "real" interaction of the patient in the virtual scenery. To graduate the levels of difficulty of the "claustrophobic" environment, two different settings were created: the first was a house and the second was a lift. In 
each one of them several different environments existed which permitted the construction of performance hierarchies with degrees of increasing difficulty. We now describe them in some detail.

\subsubsection{Setting one: the house}

1) A room of $4 \times 5 \mathrm{~m}$. This room has an exit door to a external terrace or a little garden of $2 \times 4 \mathrm{~m}$. In this room there is also a large window with a blind. When the door and the window are open, a blue sky can be seen and the sound of the birds can be heard (see Figure 1 on the $\left.\mathrm{CDROM}^{1}\right)$. The door, the window and the blind can be opened and closed in three stages (see Figure 2, Figure 3, and Video clip 1). In this same room is another door to a second room or second claustrophobic setting (see Figures 4 and 5).

2) This second room measures $3 \times 3 \mathrm{~m}$. There are neither furniture nor windows, and the ceiling and floor are darker with a wood texture to give a greater sensation of closure. In the center of the room there is a lectern with several buttons by means of which the patient can interact with the virtual environment. The door of this room can be closed by the patient in three stages and, once closed, it can be blocked if he/she decides to do so by means of one of the buttons situated on the lectern (see Figures 6 and 7).

Finally, there is a wall in the room which can be displaced by the patient, making a loud noise (also with different possibilities of advance or closing by means of one of the buttons which is situated on the lectern) and keeping the patient enclosed in $1 \mathrm{~m}$ wide space (see Figures 8 and 9, and Video clip 2).

\subsubsection{Setting two: the lift}

1) In this setting there is a wide entry where with a large window. This large window can not be opened but through it one can see plants outside. From this entry the patient has access to the lift by pressing a button (see Figure 10 ).

2) The lift is designed to offer different possibilities of the claustrophobic threat by taking into account various parameters (size, placing and possibility of blocking the lift):

2.1) The lift is situated on the ground floor, large in size ( $1 \mathrm{~m}$ $\mathrm{x} 2 \mathrm{~m}$ ) and with the door open. The patient can be inside the lift with the door open and looking at the space of the entry. He can enter and exit whenever he wants (see Figure 11).

2.2) The lift is closed and working. The patient presses a button and the lift closes and starts working (see Figure 12 and Figure 13). The lift can move to different floors and the patient can get off on any floor.

2.3) The lift is blocked. The patient can block the lift by pressing a button and, from that moment, he will not be able to get out in any way during a period of time, which is random and predetermined by the system. The aim is to simulate an average.

2.4) A small-sized lift in which one of the walls advances with a loud noise and encloses the patient in a space of $1 \mathrm{~m} \mathrm{x}$ $1 \mathrm{~m}$ (see Figure 14). All the possibilities allowed in the big lift are also able to be realized in the small lift.

\subsection{TECHNICAL ASPECTS}

Initially we used a Silicon Graphics Indigo High Impact computer graphics workstation as hardware. Then in order to extend a wide commercial use of this technology, we created a virtual reality system used in clinical psychology adaptable to an inexpensive PC. For the present study a Pentium Pro computer with 128M of RAM and an AccelEclipse graphic card with $32 \mathrm{Mg}$ was used as the hardware platform. The operating system was Windows NT and the virtual reality program was World Up (Sense8). VR peripherals included a V6 HMD (Virtual Research) and a 3D mouse.

Virtual reality technology provides a new interactive form between user and computer where the user is not a mere outside observer of images in a monitor, but an active participant inside the 3D images, called virtual scenes or virtual environments (VE). He/she can interact with the objects of the scene. We think that this interaction can be more effective due to the incorporation of technical methods — algorithms — which allow images to have a greater quality of visual realism. The algorithms are not included in the typical virtual reality applications software. The use of global illumination techniques — radiosity — in the development of virtual environments also improves the level of realism.

To obtain realistic solutions, radiosity solutions of the VE were calculated by using LightScape v.3.0 software (LightScape Technologies) and textures generated by the radiosity solutions were mapped on the geometry of the VE.

\subsubsection{RADIOSITY AND VIRTUAL REALITY}

With the introduction of raster display technology, several simple Local Illumination Models were developed for displaying computer generated images of surfaces, in particular the Gouraud [Goraud, 1971] and Phong models [Phong, 1975]. These algorithms use local illumination models which depend only on the characteristics of the surface in question and the light sources; information regarding other objects in the environment is ignored. The Phong and Gouraud models assume all light sources to be infinitely small, with isotropic intensity distributions. When computing illumination effects using local shading models, no information about the position and characteristics of other surfaces in the environment are taken into account: objects appear shaded as if they were floating in empty space. Complex (but often necessary) illumination effects such as shadows and accurate interreflections are not 
modeled. Many early works using local illumination were directed towards interactivity, and the benefits of these models are demonstrated by their numerous modern hardware implementations, enabling the display of hundreds of thousands of shaded polygons per second. This hardware speed-up is used in virtual reality applications, resulting in real time image refresh ratios (20-25 image/second). Unfortunately, because they are not based on concrete physical processes and quantities, these models do not produce realistic results. The appearance of images can be improved by using techniques such as texture mapping and bump mapping which modify the reflectivity and normal of a surface respectively, but even so, local illumination models fall far short of being visually realistic.

To model complex illumination effects such as shadowing and interreflections, global illumination models are needed. The two most common global illumination models are ray tracing and radiosity. Complex illumination effects are best simulated by the radiosity method. This method is capable of modeling the interreflections of light between diffuse surfaces, and can generate effects such as color bleeding (light reflecting off a colored surface) and penumbral shadows.

Visual realism of radiosity images has been tested by Meyer et al. [Meyer, Rushmeier, Cohen, Greenberg and Torrance, 1986] on static images, showing that in most cases observers detected no difference between real images and radiosity images. Unfortunately, this visual realism comes at a price. Radiosity is a notoriously computationintensive task with solutions for complex environments often taking many hours or even days to generate. Recent research has focused on algorithmic improvements with the aim of generating fast radiosity solutions without compromising accuracy. The most beneficial of such improvement was the introduction of hierarchical algorithms [Hanrahan, Salzman and Aupperle, 1991]. Recent proposals involve clustering techniques applied to radiosity solutions [Smits, Arvo and Greenberg, 1994]. These approximate radiosity solutions enable complex environments to be calculated in a more reasonable time.

Radiosity is a view-independent technique because illumination is calculated over the entire environment, rather than only for the surfaces in direct view of the camera. Thus after calculating a radiosity solution, images from various viewpoints can be displayed interactively on a high performance graphic workstation. This brings the possibility of using recent virtual reality techniques to immerse the user in a very realistically illuminated virtual environment [Airey, Rohlf and Brooks, 1990].

To introduce radiosity into virtual environments no special algorithm had been used, but a standard radiosity program, Lightscape v3.0, was employed for its efficiency in catching scenery textures with radiosity. Later, these textures were organized into the geometry of virtual environment.

The following steps have been completed: a) Given an environment, one applies the radiosity program LightScape v. 3.0 (Lightscape Technologies).

b) Taking the result of the previous application of radiosity program, one catches the desired textures. LightScape v. 3.0 (Lightscape Technologies) has the "Mesh To Texture" option. With this option the user can select, within the environment resulting from the application of the radiosity algorithm, the surfaces to which he/she wants to apply texture. The process takes place through a succession of pages within a dialog, which lets you make choices to determine how the conversion will be done.

c) The extracted textures are put into the corresponding geometries of the virtual environments using World $U p$ (Sense8).

Then the advances obtained with this method should be apparent in the visual realism of virtual environments.

In the images of Figures 1 though 14 the visual appearance is rather poor, since programs of virtual environments like WorldUp (Sense8) have low image quality. Nevertheless, it is possible to make use of certain technologies to obtain shades (Figures 10 and 11) in order to improve visual realism. But as we can see in the image of Figure 15, the realism obtained with the preceding method is better.

\section{Conclusions}

As we have pointed out previously, we have already probed the effectiveness of this software, and we can state that the claustrophobic context is able to activate a high degree of anxiety in the patients and that, through virtual exposure, is effective in overcoming the phobia. Moreover, this result was achieved by using virtual exposure as a unique technique, that is, with no combination of other psychological treatment techniques. Therefore VR appears to be very useful in a therapeutic perspective [Botella, Baños, Perpiñá, Villa, Alcañiz y Rey, 1998; Botella, Perpiñá, Baños, Ballester y Quero, in press].

We would not like to finish the therapy without "going back into reality". We have defended the possible contributions that VR may have in the field of psychological treatments. However, as we pointed out at the beginning of this work, we should not confuse facts and fiction, and the available facts point out the convenience of taking into account the clear limitations which, up to now, the new tool has been presenting.

1. The virtual world is still rudimentary. It reminds us of the cinema at its beginnings. Those first films, jerkey and without sound, have a certain similarity to these simple and still quite artificial textures. More work is necessary to achieve a higher degree of realism and determine which psychological factors or other kind of factors have influenced the "reality judgement" which the person realizes. 
2. As pointed out before, we think that it is fundamental to go on working in order to achieve more effective ways to introduce the "self" into the virtual world.

3. VR also has clear limits. Although you can "live it" lots of times, it is only "an adventure" from which you always come back into the "real world", and it may be hard "to come back". Taking this into account, it also appears necessary to limit the possible harmful effects of VR misuse.

4. Associated with the previous point, it has been pointed out that virtual walks may produce secondary effects, basically disorientation and neurovegetative symptoms (sickness, nausea, etc.), and, to a lesser extent, some ocular-motor perturbation. However, methods minimizing and controlling these alterations have been developed [Stanney and Kennedy, 1997]. The problems do not always appear. The lack of negative collateral effects has been reported in an explicit way a [Riva et al., 1997]. This is a topic that is being submitted to controlled research at this moment [Viire, 1997]. We hope that these efforts, in a short time, will provide a way for future systems to be considered as completely "safe".

5. It is necessary to collect further data regarding the effectiveness as a sole tool versus its effectiveness used in conjunction with other common therapeutic procedures, such as "in vivo" exposure or imagination exposure.

6. It is still an expensive tool, although if it proves itself and the resulting demand is high, the price might drop.

7. It is a new tool and practically all the work has yet to be done. A fundamental aspect of future work is to construct a theoretical frame that permits predictions and organization of the results.

In our opinion VR has a great future, and the applications that have appeared up to now are only the beginning of an enormous development. As we previously mentioned, it is difficult to think of an application, which cannot be created. It is only a matter of time and money. The important points are then (1) In which fields should we work?, (2) Which applications make more sense? and (3) Which applications are more useful, have more impact, or benefit more people? [Inman et al., 1997]. We are all affected by the challenge of what kind of psychological cyberspace is better to create.

\section{Acknowledgements}

This project was partially sponsored by the Consellería de Cultura, Educaciò i Ciència (Generalitat Valenciana) (GV-D-ES-17-123-96) and the Instituto de la Mediana y Pequeña Empresa de la Generalitat Valenciana (IMPIVA) (971801003559).

\section{REFERENCES}

Airey, J.M., Rohlf, J.H.\& Brooks, F.P. (1990). Towards Image Realism with Interactive Update Rates in Complex Virtual Building Environments. In Computer Graphics. Symposium on Interactive 3D Graphics) $24: 2$ (March 1990), pp. 41-50.

Barlow, D.H. (1988). Anxiety and its disorders: The nature and treatment of anxiety and panic. New York: Guilford Press.

Booth, R. \& Rachman, S. (1992). The reduction of claustrophobia-I. Behaviour Research and Therapy, 30, 207-221.

Botella, C.; Baños, R.M.; Perpiñá, C.; Villa, H; Alcañiz, M. \& Rey, A. (1998). Virtual reality treatment of claustrophobia: A case report. Behaviour Research and Therapy.

Botella, C.; Perpiñá, C., Baños, R.M., Ballester, R. \& Quero, S. (in press). El tratamiento de la claustrofobia mediante técnicas de Realidad Virtual. Análisis y Modificación de Conducta.

Carlin, A.S., Hoffman, H.G. \& Weghorst, S. (1997). Virtual reality and tactile augmentation in the treatment of spider phobia: a case report. Behaviour Research and Therapy, 35, 153-158.

Gouraud, H. (1971). Illumination for Computer Generated Pictures. In Communications of the ACM 18:60, pp. 311317.

Greenleaf, W. (1995). Rehabilitation, Ergonomics and disability solutions using virtual reality technology. En A. Morgan y R. Satava (Eds.) Interactive technology and the new paradigm for healthcare.. IOS Press, Washington. 415-422.

Hanrahan, P., Salzman, D. \& Aupperle, L. (1991). A Rapid Hierarchical Radiosity Algorithm. In Computer Graphics (SIGGRAPH' 91 Proceedings) 24:4, pp. 197 206.

Inman, D. Loge, K. \& Leavens, J. (1997). Virtual reality and rehabilitation. Communications of the ACM, 40, 5358.

Knox, D., Schacht, C. \& Turner, J. (1993). Virtual reality: A proposal for treating test anxiety in college students. College Student Journal, 27, 294-296.

Korchin, S. \& Sands,S. (1983). Principles common to all psychotherapies. En C. E. Walker (Ed.) The handbook of clinical psychology. New York: Dow Jones-Irwin.

Meyer, G.W., Rushmeier, H.E., Cohen, M.F., Greenberg, D.P.\& Torrance, K.E. (1986). An Experimental Evaluation of Computer Graphics Imagery. In $A C M$ Transactions of Graphics 5:1, pp. 30-50.

North, M. \& North, S. (1994). Virtual environments and psychological disorders. Electric Journal of Virtual Culture. 2, (4) 25-34.

North, M. \& North, S. (1996). Virtual reality psychotherapy. The Journal of Medicine and Virtual Reality, 1, 28-32.

North, M. North, S. \& Coble, J. (1997). Virtual reality therapy. Ann Arbor, Michigan. I.P.I. Press. 
Öst, L-G. (1987). Age of onset in different phobias. Journal of abnoraml Psychology, 96, 223-229.

Phong, B.T. (1975). Illumination for Computer Generated Pictures. In Communications of the ACM 18:6 (1975), pp. 311-317.

Riva, G., Melis, I. \& Bolzoni, M. (1997). Treating Bodyimage disturbances. Communication of the ACM, 40, 6971.

Rothbaum, B. Hodges, L.. Kooper, R. Opdyke, D. Williford J. \& North, M. (1995a). Effectiveness of computer generated (Virtual reality) graded exposure in the treatment of acrophobia. American Journal of Psychiatry, 152, 626-628.

Rothbaum, V. O., Hodges, L. F., Kooper, R., Opdyke, D., Williford, J. S. \& North, M. (1995b). Virtual-Reality Graded Exposure in the Treatment of Acrophobia - A Case Report. Behaviour Therapy, 26 (3), 547-554.

Rothbaum, V. O., Hodges, L., Watson, B. A., Kessler, G. D. \& Opdyke, D. (1996). Virtual-Reality Exposure Therapy in the Treatment of Fear of Flying - A Case-Report. Behaviour Research and Therapy, 34 (5-6), 477-481.

Schneider, J. (1982). Lens-assisted in vivo desensitization to heights. Journal of Behaviour Therapy and Experimental Psychiatry, 13 (4) 333-336.

Smits, B.E., Arvo, J.R.\& Greenberg, D.P. (1994). A Clustering Algorithm for Radiosity in Complex Environments. In Computer Graphics Proceedings, Annual Conference Series, ACM SIGGRAPH, pp. 435442.

Stanney, K. \& Kennedy, R. (1997). The psychometrics of the Cybersickness. Communications of the ACM, 40, 6768.

Strickland, D., Hodges, L., North, M. \& Weghorst, S. (1997). Overcoming phobias by virtual exposure. Communications of the ACM, 40, 34-39.

Tart, C. T. (1991). Multiple personality, altered states and virtual reality: the world simulation process approach. Dissociation, 3, 222-233.

Viire, E. (1997). Health and safety issues for virtual reality. Communications of the ACM, 40, 40-41.

Vince, J. (1995). Virtual reality systems. Cambridge. ACM Press.

\section{BIOGRAPHY}

Dr. Cristina Botella Arbona, the lead author, has been a psychologist since 1978 (University of Valencia). She received her $\mathrm{PhD}$ at the University of Valencia in 1983. She became a Senior Lecturer at the University of Valencia in 1986. Then in 1992 she became Professor (Psychological treatments) at Jaume I University. She has been head of the Psychological Assistance Service at the Jaume I University since 1993. Her research has focused on psychological treatments for emotional disorders. She currently heads the "PREVI" research group dedicated to the study of VR applications to the treatments of psychological problems such as claustrophobia, agoraphobia, fear of flying, and body image disturbances in eating disorders.

Contact information:

C. Botella.

Departamento de Psicología Básica

Clínica y Psicobiología

Carretera de Borriol, s/n

12080-Castellón

SPAIN

FAX: 34-964-345751

Email: Botella@psb.uji.es 